MATEC Web of Conferences 33, 05007 (2015)

DOI: $10.1051 /$ matecconf/ 20153305007

(C) Owned by the authors, published by EDP Sciences, 2015

\title{
Stress-induced transformation in a Ni-Mn-In alloy and the concomitant change of resistivity
}

\author{
V.V. Kokorin ${ }^{1}$, S.M. Konoplyuk ${ }^{1, a}$, A. Dalinger ${ }^{2}$, S. Thürer ${ }^{2}$, G. Gerstein², A.Mashirov ${ }^{3}$, Yu.P. Stetskiv ${ }^{1}$, H.J. Maier ${ }^{2}$ \\ ${ }^{1}$ Institute of magnetism of NASU and MESU, Vernadsky blvd., 03680, Kyiv, Ukraine \\ ${ }^{2}$ Institut für Werkstoffkunde (Materials Science), Leibniz Universität Hannover, An der Universität 2, D - 30823 Garbsen, Germany \\ ${ }^{3}$ Kotelnikov Institute of Radioengineering and Electronics of RAS, Mokhovaya 11-7, Moscow, 125009, Russia
}

\begin{abstract}
In this work, the influence of mechanical stress on magnetic properties and electric resistance of a Ni-MnIn alloy was studied. It is shown that compression of Ni-Mn-In polycrystalline specimens brings about a stressinduced martensitic transformation. Optical images recorded in-situ confirmed the formation of a martensitic structure during loading and back-transformation upon unloading. Unloading after deformation of specimens that had experienced compressive strains up to $6 \%$ resulted in full recovery of their resistivity and magnetic susceptibility. The sharp increase in the electric resistance caused by the stress-induced transformation opens up new possibilities for NiMn-In alloys to be used as a material for sensors responding to mechanical stress.
\end{abstract}

\section{Introduction}

Investigations in the field of shape memory and superelastic alloys quite often have aimed at developing alloys with improved performance to widen their prospective applications. In the beginning, functional properties of shape memory alloys were typically associated with the thermoelastic martensitic transformation driven by temperature and mechanical stress application. In the last decades, Heusler-type Ni$\mathrm{Mn}-\mathrm{Ga}$ alloys were found to show magnetically driven shape memory deformation attributed to the large magnetocrystalline anisotropy of the martensitic phase [1]. Further investigations on Heusler compounds resulted in the discovery of alloys with a martensitic transition between a high-temperature ferromagnetic phase and low-temperature phase with weak magnetism. These materials are called metamagnetic alloys and include Mn-rich Ni-Mn-X (X=In, Sn, Sb) based alloys [24]. Their distinctive feature is that the martensitic transition in these compounds can be shifted toward lower temperatures by $2-11 \mathrm{~K}$ in a magnetic field of $1 \mathrm{~T}$ [5] due to a strong coupling between the magnetic and crystallographic structure. This provides for an opportunity to use them not only in actuator applications triggered by a magnetic field but also in magnetic refrigeration where the latent heat is absorbed during the magnetically-induced phase transformation.

Recent studies have demonstrated that in addition to the giant magnetocaloric effect [6] and giant magnetoresistance [7], the giant barocaloric effect [8] is intrinsic to metamagnetic alloys as well.
The origin of these effects in Ni-Mn-X (X=In, Sn, Sb) compounds lies in their ability to change the characteristic transformation temperatures in response to a variation of external parameters such as a pressure, magnetic field etc. Up to now, most studies mainly focused on the caloric properties but physical effects associated with a change of electric and magnetic properties during stress-induced martensitic transformation can be anticipated in these alloys as well. In the present study, a Ni-Mn-In alloy was employed as this system shows a more pronounced change of electric resistivity and magnetization due to the martensitic transition as compared to $\mathrm{Ni}-\mathrm{Mn}-\mathrm{Sb}$ and $\mathrm{Ni}-\mathrm{Mn}-\mathrm{Sn}$ systems.

\section{Materials and methods}

An ingot was prepared by induction melting in an argon atmosphere followed by homogenization at a temperature of $1223 \mathrm{~K}$ for 50 hours and an additional annealing at $1023 \mathrm{~K}$ for 50 hours. The composition of the alloy obtained was determined with energy dispersive x-ray spectroscopy as $\mathrm{Ni}_{45.4} \mathrm{Mn}_{40} \mathrm{In}_{14.6}$ (at.\%). Samples for compression tests with square cross section of $2 \mathrm{~mm} \times 2$ $\mathrm{mm}$ and length of $4 \mathrm{~mm}$ were spark cut from the ingot. Electrical resistance was measured by the four-probe method and low-field magnetic susceptibility was determined by the induction method. The temperature of samples during measurements was varied at a rate of 5 $\mathrm{K} / \mathrm{min}$.

A miniature mechanical load frame was used for the compression test. The samples were loaded along their

\footnotetext{
a Corresponding author: ksm@imag.kiev.ua
} 
long geometrical axis and the strain was measured along the same direction. A Keyence VHX 1000D optical microscope with a VHZ 100 UR (100-1000 magnification) lens was employed for observation of the microstructure.

\section{Results and discussion}

Heusler alloys are very sensitive to changes in chemical composition, and, in particular, the characteristic phase transition temperatures are affected. The electric and magnetic properties follow the structural and magnetic changes during the martensitic transformation allowing for an accurate determination of the characteristic temperatures. The magnetic susceptibility is very sensitive since the martensitic transformation in Ni-MnIn occurs between phases with quite different magnetism [9]. Fig. 1a shows the variation of magnetic susceptibility with temperature. On cooling, ferromagnetic ordering appears when the temperature reaches $320 \mathrm{~K}$ (Curie point). An additional decrease of the sample temperature leads to the onset of the martensitic transformation, which is accompanied by a drop of the magnetic susceptibility.

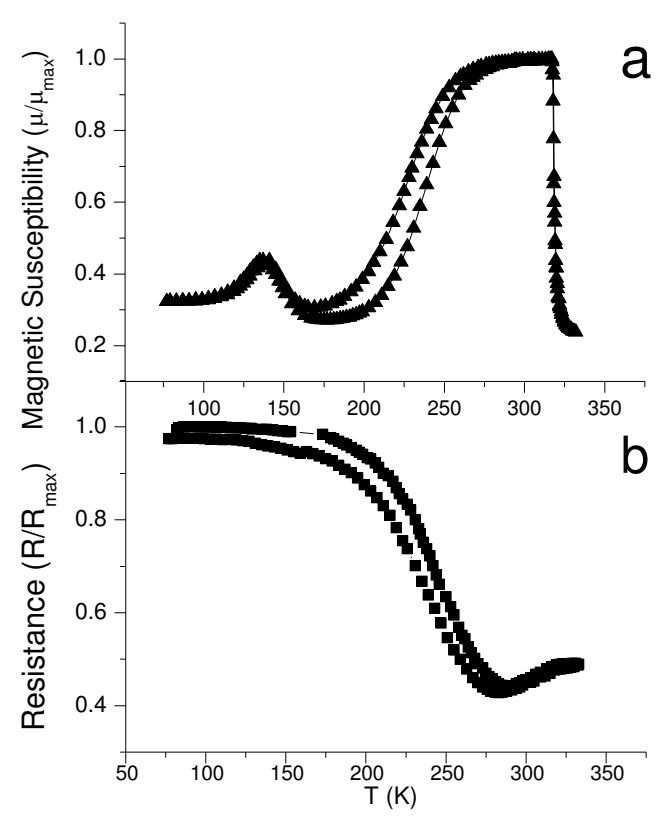

Figure 1. a) Magnetic susceptibility and b) electrical resistance of $\mathrm{Ni}_{45.4} \mathrm{Mn}_{40} \mathrm{In}_{14.6}$ sample as a function of temperature, recompiled from [10].

From Fig. 1, $M_{\mathrm{S}}=270 \mathrm{~K}$ (martensite start temperature), $M_{\mathrm{f}}=195 \mathrm{~K}$ (martensite finish temperature), $A_{\mathrm{S}}=205 \mathrm{~K}$ (austenite start temperature), and $A_{\mathrm{f}}=275 \mathrm{~K}$ (austenite finish temperature).

It is well known that the low-temperature phase in Ni-Mn-In system is a low-symmetry crystallographic phase with mixed ferromagnetic and antiferromagnetic interactions $[9,11]$. In an Mn-rich alloy, the excess of Mn atoms relative to the stoichiometric composition results in a partial occupation of the In atomic positions by $\mathrm{Mn}$ atoms, which are located at closer distances to the other $\mathrm{Mn}$ atoms than in regular sites. The lattice parameter change and decrease in specific atomic volume associated with the martensitic transformation results in an antiferromagnetic exchange between these atoms. Indeed, Fig. 1a shows a peak at a temperature of about $130 \mathrm{~K}$ that can be attributed to freezing of the magnetic moments of nano-sized ferromagnetic clusters embedded in an antiferromagnetic matrix, in a way similar to formation of a superspin glass state [12].

The resistivity plot (Fig. 1b) shows no features in this temperature region supporting the only magnetic nature of the peak. Upon the martensitic transformation the electric resistance is more than doubled. This is a very important feature as this can provide for great opportunity for new applications based on resistivity changes of the alloy.

Figure 2 demonstrates the stress-strain response of the sample at room temperature. As is seen in Fig. 2a,

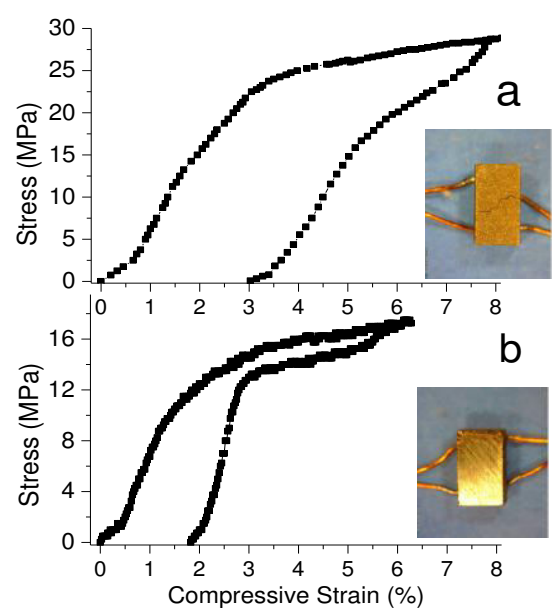

Figure 2. Stress-strain curves of $\mathrm{Ni}_{45.4} \mathrm{Mn}_{40} \mathrm{In}_{14.6}$ specimens a) compressed to a strain of $8 \%$ and b) compressed about $6 \%$ [10]. The inset shows the specimens after the tests.

deformation up to $8 \%$ is accompanied by a stressinduced martensitic transformation, which gives rise to the plateau-like portion in the curve. The phase transformation begins as the stress and the corresponding strain achieve about $24 \mathrm{MPa}$ and $3 \%$, respectively. Below this stress level, the austenitic phase deforms only elastically. Only a part of the total deformation is recoverable upon the removal of the stress. The inset in Fig. 2a shows a crack across the post-stressed sample, which is responsible for apparent irrecoverable strain.

In another test, the maximum strain was limited to 6 $\%$ (Fig. 2b). In contrast to Fig. 2a, the critical stress for the onset of the martensitic transformation is lower. The difference can be explained based on the slight inhomogeneity of the ingot that results in different characteristic phase transformation temperatures, and thus, different critical stress levels. In this test no large cracks are evident on the surface of the specimen, although the residual strain after unloading was about 1.7 $\%$.

The stress-strain response was recorded simultaneously with the variation of magnetic 
susceptibility and electric resistance (Fig. 3). The level of critical strain (3-3.5\%), where these start to change sharply, coincides with the onset of the martensitic transition in the stress-strain curves (Fig. 2). Similar to the thermally-induced transformation (Fig. 1), the magnetic susceptibility drops and the resistance increases during the stress-induced transformation. Clearly, the resistivity also responds to structural changes resulting from cracking. For instance, upon unloading, the electric resistance of the specimen that was compressed up to 8 $\%$ strain, first starts decreasing but then as the cracks develop it begins to increase (Fig. 3b).

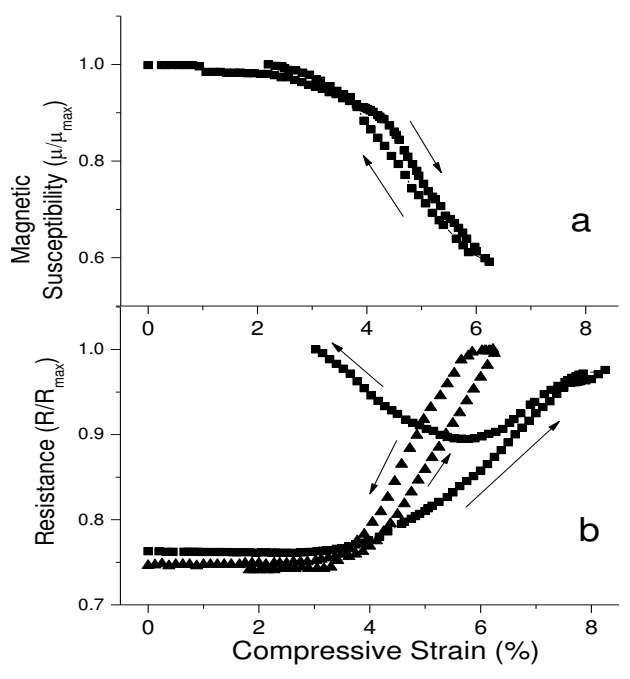

Figure 3. Variation of a) magnetic susceptibility and b) electric resistance of $\mathrm{Ni}_{45.4} \mathrm{Mn}_{40} \mathrm{In}_{14.6}$ samples with strain. Partly recompiled from [10].

In case of smaller deformation, the loading-unloading cycle is accompanied by a fully reversible change of both the resistivity and the magnetic susceptibility. This is an evidence of superelastic behaviour. It is worth noting that the change of these properties under the applied stress only accounts for a small part of their total possible variation due to the phase transformation (Fig. 1).

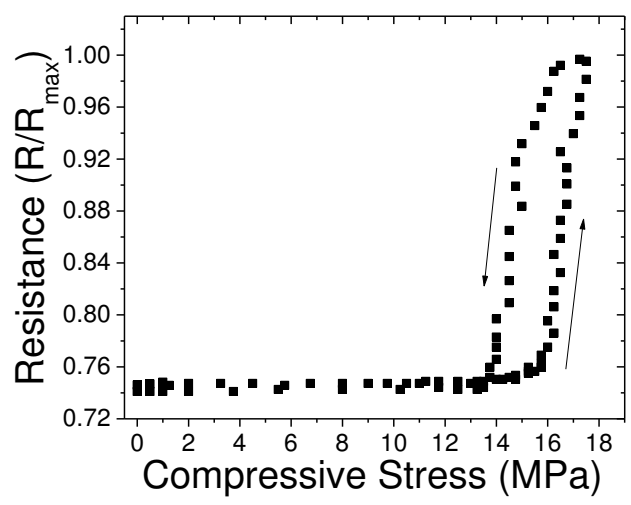

Figure 4. The electric resistance of $\mathrm{Ni}_{45.4} \mathrm{Mn}_{40} \mathrm{In}_{14.6}$ sample as a function of the compressive stress [10].
The formation of the stress-induced martensite involves a very abrupt change of the resistivity (Fig. 4). As soon as the applied stress reaches $16 \mathrm{MPa}$, an increase in the electrical resistance by more than $30 \%$ occurs. Upon unloading, the resistivity returns to the initial level. Apparently, this elastoresistive effect, i.e. reversible change of the resistivity occurs in accordance with the change in the volume fraction of transformed martensite. Further mechanical loading can increase the magnitude of this effect up to $150 \%$ (Fig. 1). However, the brittleness of the polycrystalline samples will probably require the use of single crystals or heavily textured polycrystals to fully exploit this effect in the Ni-Mn-In alloy.

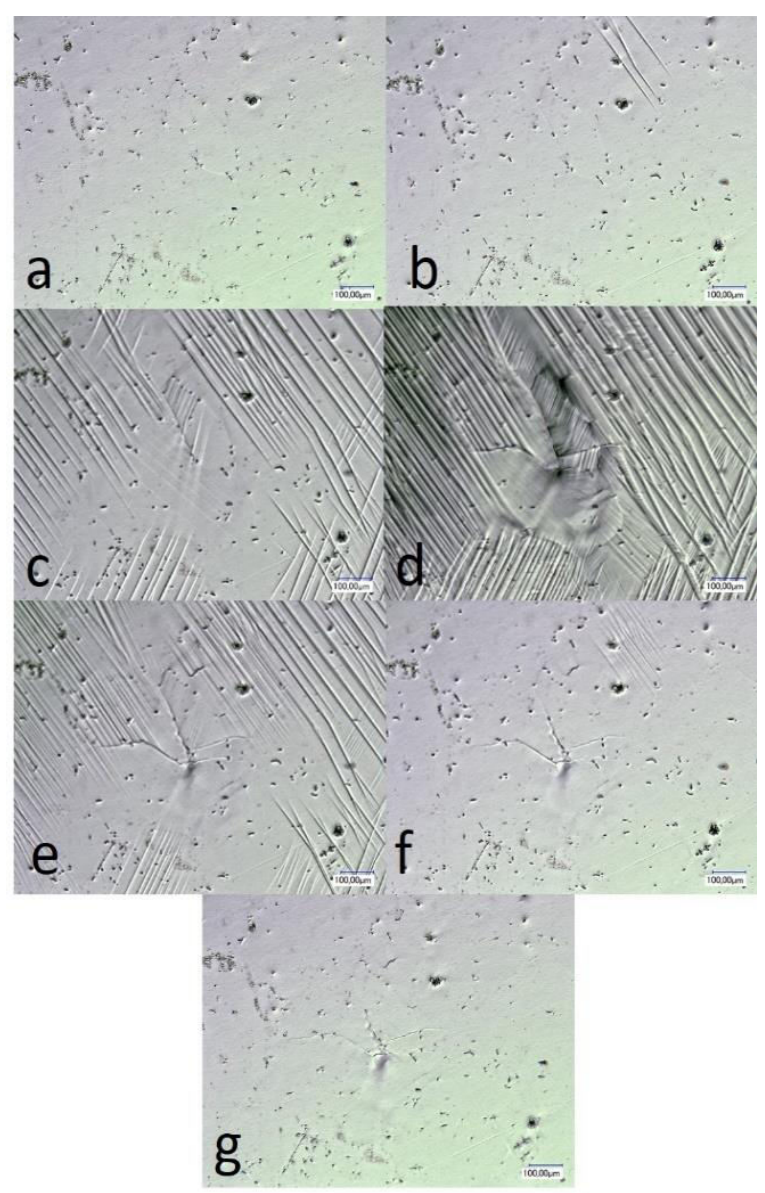

Figure 5. Microstructural changes during the stress-induced transformation in a $\mathrm{Ni}_{45.4} \mathrm{Mn}_{40} \mathrm{In}_{14.6}$ sample. Compressive strain upon loading: a) $0 \%$, b) $1.9 \%$, c) $3.25 \%$, d) $5 \%$, and upon unloading: e) $3.25 \%$,f) $1.9 \%$, g) $0.25 \%$. After [10].

In order to shed light on the corresponding structural changes occurring in the process of the stress-induced transformation, microstructural observations were performed. In these tests, the maximum deformation was limited to $5 \%$ strain to avoid premature fracture of specimen. Figure 5 shows a sequence of images recorded from the onset of compressive loading to full unloading. The first martensitic plates appear at a strain of about $2 \%$ (Fig. 2b). This strain value corresponds to the linear 
elastic region in the stress-strain plot. Subsequently, the increase of the applied stress leads to massive formation of martensite with different variants over a strain range from 3 to $5 \%$. This regime corresponds to the flat part of the stress-strain curve and the distinct change of the properties examined in this study. Further compression, however, leads to nucleation of cracks along the boundary between grains with different orientations.

The cracks remain upon unloading (Fig. 5g). The nucleation and growth of cracks is a result of the build-up of intergranular local stresses in the polycrystalline samples. The stresses arise due to the growth and reorientation of martensitic variants inside grains as a response to mechanical loading. Some grains are oriented unfavourably with respect to the external stress. This leads to a difference in the fraction of martensite formed inside the individual grain, and thus, to different transformation strains of the differently oriented grains. Eventually, the interfaces and boundaries between these grains become preferred places for crack nucleation and growth.

The martensite transforms back into austenite as the applied stress decreases and finally disappears at a strain below $2 \%$. After completion of this test the residual macroscopic strain was $0.25 \%$, which is mainly a result of the formation of the small cracks.

\section{Conclusions}

1. The superelastic deformation of a $\mathrm{Ni}_{45.4} \mathrm{Mn}_{40} \mathrm{In}_{14.6}$ polycrystalline alloy yielded $6 \%$ strain due to the martensitic transformation. An additional increase of the applied stress leads to large residual strains and a non-reversible change of properties.

2. The martensitic transformation in compression at strains below $6 \%$ is accompanied by large reversible changes of the resistivity and the magnetic susceptibility. The change of the electric resistance under an applied stress of about $16 \mathrm{MPa}$ (elastoresistive effect) reached $30 \%$ and may be increased in single crystals.

3. The microstructural observations confirmed the superelastic character of deformation but also demonstrated that small cracks nucleate early due to incompatibility between differently oriented grains. Macroscopically this results in apparent residual deformation upon unloading.

\section{References}

1. K. Ullakko, J. K. Huang, C. Kantner, R. C. O'Handley, V. V. Kokorin, Appl. Phys. Lett. 69, 1966 (1996)

2. Y. Sutou, Y. Imano, N. Koeda, T. Omori, R. Kainuma, K. Ishida, K. Oikawa, Appl. Phys. Lett. 85, 4358 (2004)

3. T. Krenke, M. Acet, E. F. Wassermann, X. Moya, L. Mañosa, A.Planes, Phys. Rev. B 73, 174413 (2006)
4. K. Koyama, T. Kanomata, R. Kainuma, K. Oikawa, K. Ihida, K.Watanabe, Appl. Phys. Lett. 88, 132505 (2006)

5. V. V. Kokorin, V. V. Koledov, V. G. Shavrov, S. M. Konoplyuk, S. Thürer, D. A. Troyanovsky, H. J. Maier, V. V. Khovaylo, J. Appl. Phys. 116, 103515 (2014)

6. Z. D. Han, D. H. Wang, C. L. Zhang, S. L. Tang, B. X. Gu, Y. W. Du, Appl. Phys. Lett. 89, 182507 (2006)

7. S. Y. Yu, Z. H. Liu, G. D. Liu, J. L. Chen, Z. X. Cao, G. H. Guang, B.Zhang, X. X. Zhang, Appl. Phys. Lett. 89, 162503 (2006)

8. L. Mañosa, D. González-Alonso, A. Planes, E. Bonnot, M. Barrio, J.-L. Tamarit, S. Aksoy, M. Acet, Nat. Mater., 9, 478 (2010)

9. S. Aksoy, M. Acet, P. P. Deen, L. Mañosa, A. Planes, Phys. Rev. B79, 212401 (2009)

10. V. V. Kokorin, S. M. Konoplyuk, A. Dalinger, S. Thürer, G. Gerstein, H. J. Maier, Appl. Phys. Lett., 106, 131908 (2015).

11. N. V. Rama Rao, V. Chandrasekaran, K. G. Suresh, J. Appl. Phys. 108, 043913 (2010)

12. S.M. Konoplyuk, V.V. Kokorin, O.V. Kolomiets, A.E. Perekos, V.M. Nadutov, J. Magn. Magn. Mater. 323, 763 (2011) 